

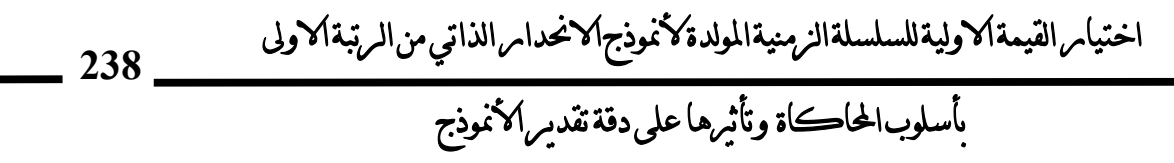

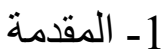

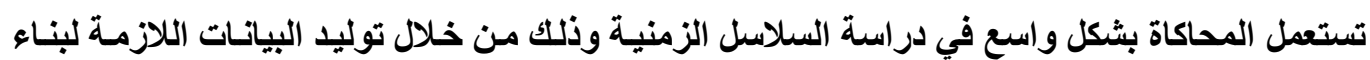

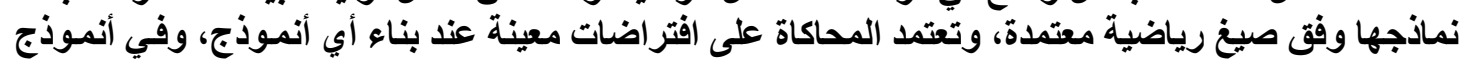

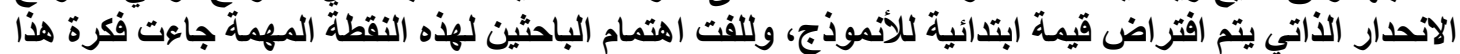

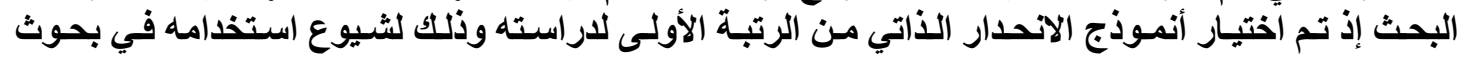

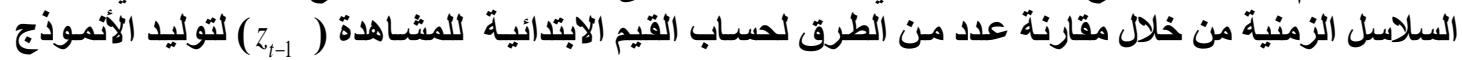

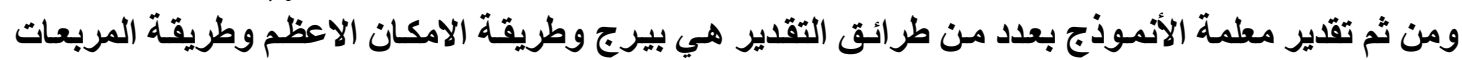

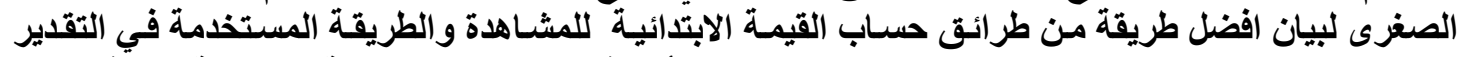

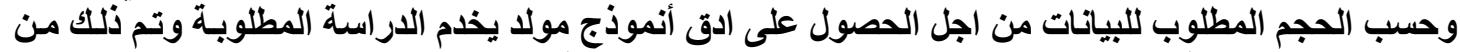

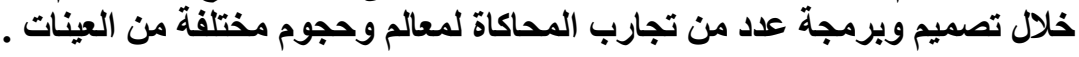

First Order Autoregressive Model

$$
\text { 2- أنموذج الانحدار الذاتي من الرتبة الاولى [2] }
$$

إنْ أنموذج الانحدار الذاتي Autoregressive Model فيه القيمة الحالية للسلسلة الزمنية يعبر عنها بدلالية

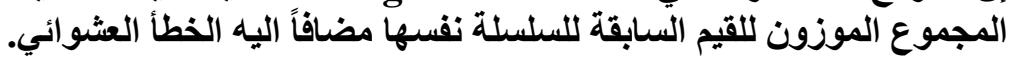

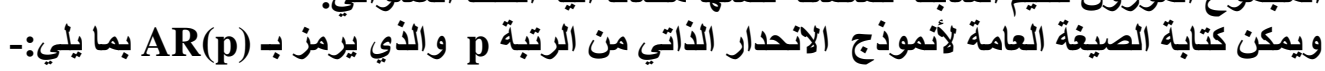
$\mathbf{t}=\mathbf{p}+\mathbf{1}, \mathbf{p}+\mathbf{2}, \quad$..... (1-1) $z_{t}=\phi_{1} z_{t-1}+\phi_{2} z_{t-1}+\ldots+\phi_{p} z_{t-p}+a_{t}$

حيث ان

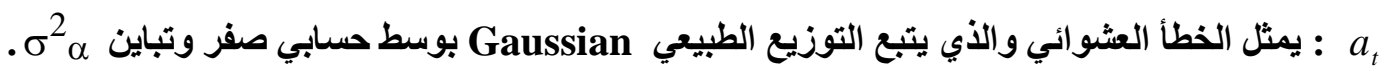

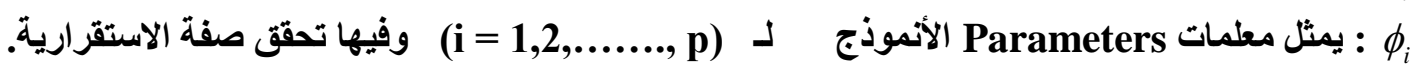
ويمكن كتابة الصيغة اعلاه على النحو الاتي:-

$$
\left(1-\phi_{1} B-\ldots . \phi_{P} B\right) z_{t}=a_{t}
$$

First Order Autoregressive Model ويمكن كتابة أنموذج الاتحدار الذاتي من الرتبة الأولى

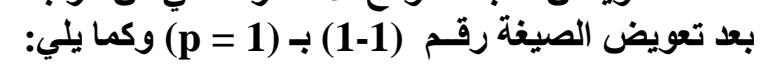

$z_{t}=\phi_{1} z_{t-1}+a_{t}$

والذي يرمز لـه بـ (1) AR(1) ويدعى أيضـا بعملية مساركوف Markov Process وباستعمال عامل الارتداد

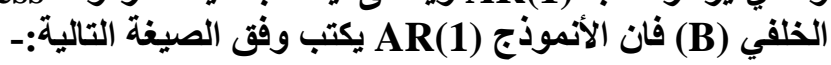
$\phi(B) z_{t}=a_{t}$ ... (1-4)

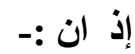
$\phi(B)=1-\phi_{1} B$ 


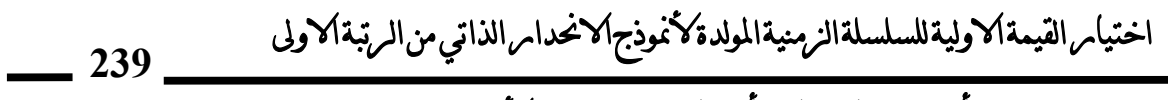

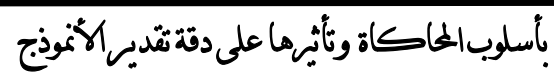

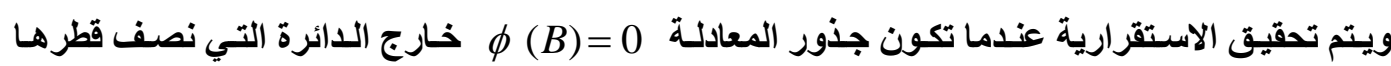

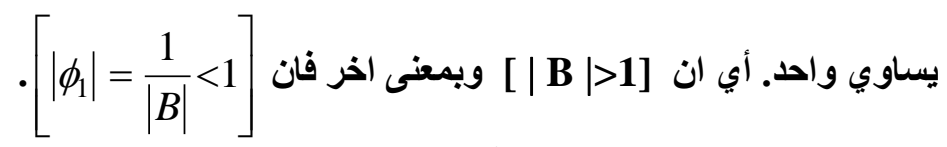

$\gamma_{\mathrm{k}}=\operatorname{cov}\left(\mathbf{Z}_{\mathrm{t}}, \mathbf{Z}_{\mathrm{t}+\mathrm{k}}\right)=\mathbf{E}\left(\mathbf{Z}_{\mathrm{t}}, \mathbf{Z}_{\mathrm{t}+\mathrm{k}}\right)$

$\gamma_{k}=\frac{\phi_{1}^{k} \sigma_{\alpha}^{2}}{1-\phi_{1}^{2}}$

$$
\gamma_{0}=\operatorname{var}\left(Z_{t}\right)=\sigma_{\alpha}^{2} \sum_{i=0}^{\infty} \phi_{1}^{2 i}=\frac{\sigma_{\alpha}^{2}}{1-\phi_{1}^{2}}
$$

ومن الصيغة اعلاه فان تباين أنموذج AR(1) كما يأتي:-

$$
\rho_{k}=\phi_{1} \rho_{k-1}= \begin{cases}\phi_{1}^{k}, & k \geq 1 \\ 1, & k=0\end{cases}
$$

وان دالة الارتباط الذاتي مساوية الى :-

3 - طرق التقدير: [4] [3]

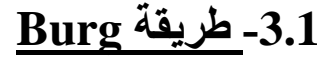

وتسمى هذه الطريقة ايضا بطريقة الوسط التوافقي Harmonic-Mean Method ويعبر عنها بالاتي

للسلسلة الزمنية (1-1) يتم تقدير معالم الأنموذج بالاعتماد على تصغير معدل تباين الأخطاء الأمامي والخلفي $\rho_{p}=\rho_{p}^{f}+\rho_{p}^{b} \quad \ldots(\mathbf{1 - 8})$

متوسط تباين الخطأ الأمامي

$$
\begin{aligned}
& \text {... (1-9) } \rho_{p}^{f}=\frac{1}{N-p} \sum_{n=p+1}^{N}\left(a_{p}^{f}(n)\right)^{2} \\
& a_{p}^{f}(n)=z(n)-\sum_{k=1}^{p}\left(\phi_{k}^{p} z_{(n-k)}\right) \\
& \text { الأخطاء الأمامية } a_{p}^{f} \\
& \text {.. (1-10) } \rho_{p}^{b}=\frac{1}{N-p} \sum_{n=1}^{N-p}\left(a_{p}^{b}(n)\right)^{2} \\
& a_{p}^{b} \\
& a_{p}^{b}(n)=z(n)-\sum_{k=1}^{p}\left(\phi_{k}^{p} z_{(n+k)}\right)
\end{aligned}
$$




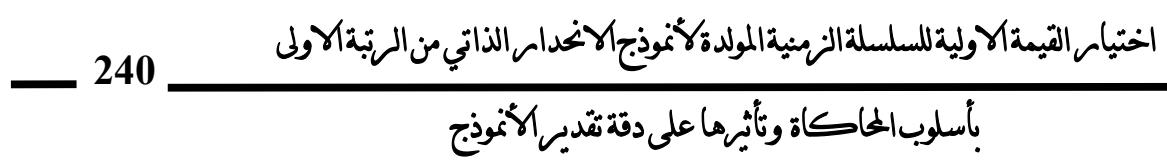

وبالاعتماد على أسلوب Levinson recursive فأن المعلمات من الرتبة p سترتبط مع المعلمات من الرتبة

$$
\begin{aligned}
& \phi_{p}(t)=\phi_{p-1}(t)-k_{p} \phi_{p-1}(p-t) \quad 1 \leq t \leq p-1 \quad \ldots \text { (1-11) } \\
& \text { وبتعويض العلاقة (1-11) في كل من (1-9) و(1-11) } \\
& a_{p}^{f}(n)=a_{p-1}^{f}(n)-k_{p} a_{p-1}^{f}(n-p) \\
& a_{p}^{f}(n-p)=a_{p-1}^{b}(n-p)-k_{p} a_{p-1}^{f}(n)
\end{aligned}
$$$$
\text { ... (1-12) } \rho_{p}=\rho_{p-1}\left(1-k_{p}^{2}\right)
$$

$$
\rho_{\mathrm{O}}=\frac{1}{N} \sum_{t=1}^{N} z(t)
$$

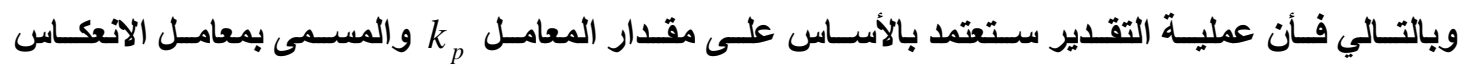
$\left|k_{p}\right| \leq 1$

وبشكل يحقق خاصية الثبات (Rtable) واي : (Rtalection Coefficient)

حيث تمكن Burg من أيجاد هذا المقدار من خلال تصغير معدل تباين الخطأ الأمامي والخلفي ليحصل على

$$
\ldots(1-13) k_{p}=\frac{2 \mathrm{E}\left[a_{p-1}^{f}(n) a_{p-1}^{b}(n-p)\right]}{\mathrm{E}\left[\left(a_{p-1}^{f}(n)\right)^{2}+\left(a_{p-1}^{b}(n-p)\right)^{2}\right]}
$$

OLS 3.2 طريقة المربعات الصغزى

$$
\begin{aligned}
& z_{t}=\phi_{1} z_{t-1}+a_{t} \\
& a_{t} \sim \operatorname{IND}\left(0, \sigma^{2} a\right) \\
& \hat{\phi}_{1}=\frac{\sum_{t=1}^{n} Z_{t-1} Z_{t}}{\sum_{t=1}^{n} Z^{2}{ }_{t-1}}
\end{aligned}
$$$$
\text { ان انموذج الانحدار الذاتي من الرتبة الاولى وفق الصيغة رقم (1-3) الآتية:- }
$$

$\sqrt{\mathrm{n}}\left(\hat{\phi}_{1}-\phi_{1}\right) \stackrel{\mathrm{L}}{\longrightarrow} \mathrm{N}\left(0,\left(1-\phi_{1}^{2}\right)\right)$

$$
\text { وفي حالة كون قيمة 1> 1 | } 1 \text { فان }
$$

(L: Converge In Law) 


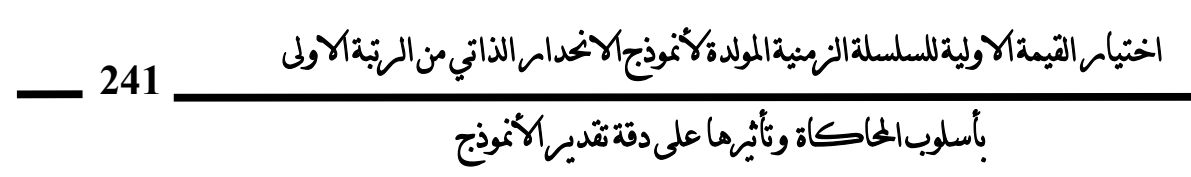

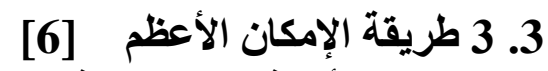

$z_{t}=\phi_{1} z_{t-1}+a_{t}$ لأنموذج الاتحدار الذاتي من الرتبة الاولى

$$
\theta \equiv\left(\phi, \sigma^{2}\right)^{\prime}
$$

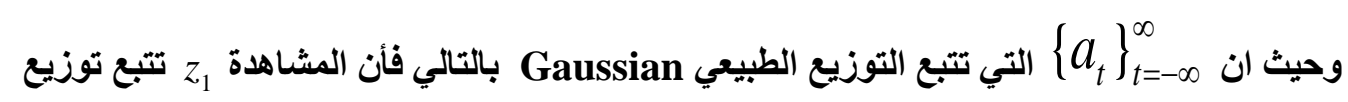
Gaussian $f_{Z_{1}}\left(z_{1} ; \theta\right)=f_{Z_{1}}\left(z_{1} ; \phi_{1}, \sigma^{2}\right)$

$$
=\frac{1}{\sqrt{2 \pi} \sqrt{\sigma^{2} /\left(1-\phi_{1}^{2}\right)}} \exp \left[\frac{-z_{1}^{2}}{2 \sigma^{2} /\left(1-\phi_{1}^{2}\right)}\right]
$$

وان

$z_{2}=\phi_{1} z_{1}+a_{2}$

$$
\begin{aligned}
& \left(Z_{2} / Z_{1}=z_{1}\right) \sim N\left(\left(\phi_{1}, z_{1}\right), \sigma^{2}\right) \quad \text { وان } \\
& \quad \ldots\left(\text { 1-15) } f_{Z_{2} / Z_{1}}\left(z_{2} / z_{1} ; \theta\right)=\frac{1}{\sqrt{2 \pi \sigma^{2}}} \exp \left[\frac{-\left(z_{2}-\phi_{1} z_{1}\right)^{2}}{2 \sigma^{2}}\right]\right. \\
& f_{Z_{2}, Z_{1}}\left(z_{2}, z_{1} ; \theta\right)=f_{Z_{2} / Z_{1}}\left(z_{2} / z_{1} ; \theta\right) . f_{Z_{1}}\left(z_{1} ; \theta\right)
\end{aligned}
$$

\section{In general}

$$
\begin{aligned}
& f_{Z_{t} / Z_{t-1}, Z_{t-1}, \ldots Z_{1}}\left(z_{t} / z_{t-1}, z_{t-2}, \ldots, z_{1} ; \theta\right) \\
& =f_{Z_{t} / Z_{t-1}}\left(z_{t-1} / z_{t} ; \theta\right) \\
& =\frac{1}{\sqrt{2 \pi \sigma^{2}}} \exp \left[\frac{-\left(z_{t}-\phi_{1} z_{t-1}\right)^{2}}{2 \sigma^{2}}\right] \\
& f_{Z_{t}, Z_{t-1}, \ldots, Z_{1}}\left(z_{t}, z_{t-2}, \ldots, z_{1} ; \theta\right) \\
& =f_{Z_{t} / Z_{t-1}}\left(z_{t-1} / z_{t} ; \theta\right) \cdot f_{Z_{t-1}, Z_{t-2}, \ldots Z_{1}}\left(z_{t-1}, z_{t-2}, \ldots, z_{1} ; \theta\right) \\
& \text { وكانت دالة الامكان } \\
& f_{Z_{T}, Z_{T-1}, \ldots, Z_{1}}\left(z_{T}, z_{T-1}, \ldots, z_{1} ; \theta\right)=f_{Z_{1}}\left(z_{1} ; \theta\right) \cdot \prod_{t=2}^{T} f_{Z_{t} / Z_{t-1}}\left(z_{t} / z_{t-1} ; \theta\right) \\
& \text { وان اللوغاريتم لاالة الإمكان هي } \\
& \text {... (1-16) } \ell(\theta)=\log f_{Z_{1}}\left(z_{1} ; \theta\right)+\sum_{t=2}^{T} \log f_{Z_{t} / Z_{t-1}}\left(z_{t} / z_{t-1} ; \theta\right)
\end{aligned}
$$




\section{2

وبالتالي فأن اللوغاريتم لدالة الإمكان لعينة مكونة من t من المشاهدات لأنموذج الانحدار الأتي من

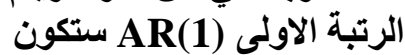
$\ell(\theta)=-\frac{1}{2} \log (2 \pi)-\frac{1}{2} \log \left[\sigma^{2} /\left(1-\phi_{1}^{2}\right)\right]-\frac{\left\{z_{1}\right\}^{2}}{2 \sigma^{2} /\left(1-\phi_{1}^{2}\right)}-[(T-1) / 2] \log (2 \pi)$

$$
\ldots(1-17)-[(T-1) / 2] \log \left(\sigma^{2}\right)-\sum_{t=2}^{T}\left[\frac{\left(z_{t}-\phi_{1} z_{t-1}\right)^{2}}{2 \sigma^{2}}\right]
$$

(Simulation) - المحاكاة 4

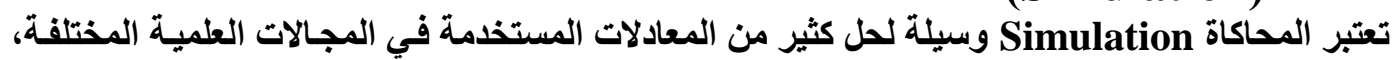

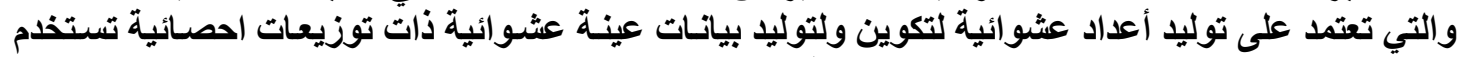

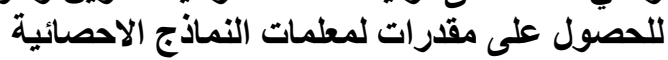

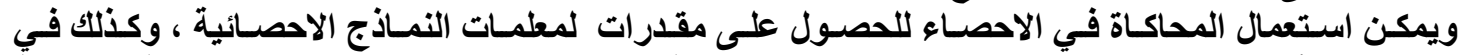

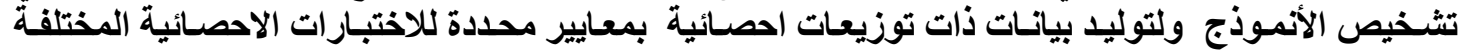

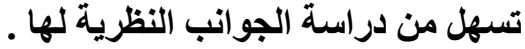
Uniform ميث تم اولا توليد أعداد عثدوائية مستقلة U(0,1) Distribution

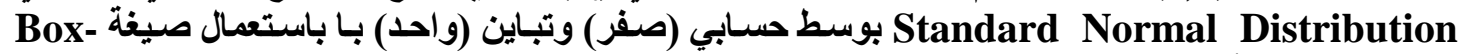
$a_{t}=\left[-2 \ln \left(u_{1}\right)\right]^{1 / 2} \cos \left(2 \pi u_{2}\right)$ $a_{t}=\left[-2 \ln \left(u_{1}\right)\right]^{1 / 2} \sin \left(2 \pi u_{2}\right)$ ومنها تم توليد أنموذج الاتحدار الذاتي من الرتبة الاولى بالصيغة (1-8) بعد حسـاب القيم الابتداية Muller للمشاهدة (

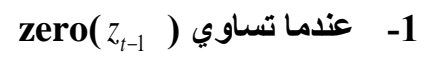

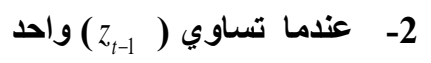

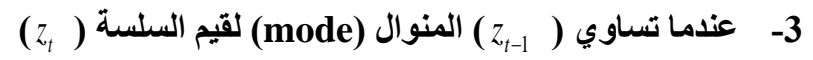
4- - علدما تساوي (

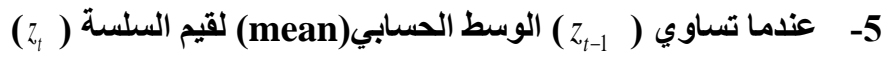

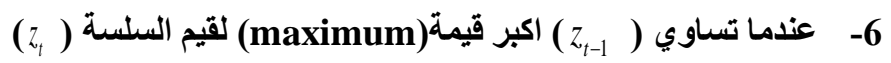

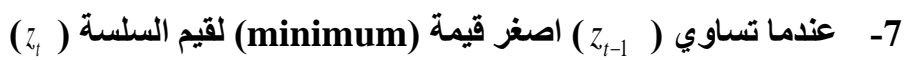

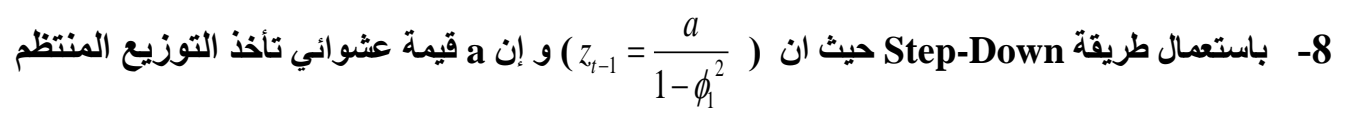

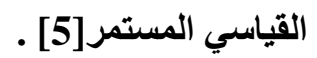

وتم استخذام عذد من طرق التقدير لتقدير معلمـة الأنموذج ولكل من طرق التوليد أعلاه بقيم مختلفة لمعلمـة

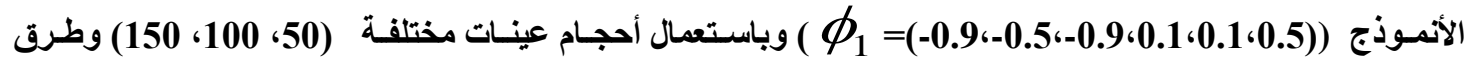
التقدير هذه هي كما يأتي :

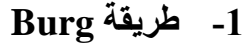

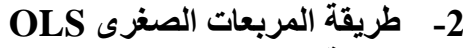

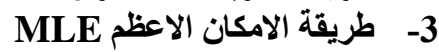

وتم تكرار جميع تجارب المحاكاة (1000) مرة لكل تجربة وتم وضع جميع النتائج في الجداول من الرقم (1) اللى (6) 


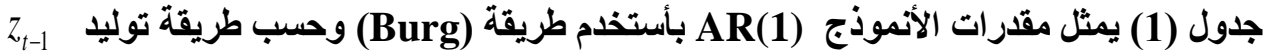

\begin{tabular}{|c|c|c|c|c|c|c|c|c|c|}
\hline$\phi_{1}$ & sample & SD & $\max$ & mean & med & $\min$ & $\bmod$ & one & zero \\
\hline \multirow{3}{*}{0.1} & 50 & 0.094662 & 0.100474 & $\mathbf{0 . 0 9 5 3 5 3}$ & 0.095485 & 0.097477 & 0.099109 & 0.092062 & 0.096056 \\
\hline & 100 & 0.095945 & 0.098477 & 0.095243 & 0.095234 & 0.101642 & 0.100599 & 0.099476 & 0.102264 \\
\hline & 150 & 0.096619 & 0.104498 & 0.102062 & 0.102069 & 0.101575 & 0.099133 & 0.099524 & 0.100011 \\
\hline \multirow{3}{*}{0.5} & 50 & 0.475488 & 0.49927 & 0.473729 & 0.473032 & 0.499771 & 0.502456 & 0.477652 & 0.472748 \\
\hline & 100 & 0.491183 & 0.500498 & 0.487763 & 0.485744 & 0.501326 & 0.506043 & 0.487234 & 0.485635 \\
\hline & 150 & 0.492707 & 0.502431 & 0.493934 & 0.491267 & 0.50209 & 0.504346 & 0.492504 & 0.491273 \\
\hline \multirow{3}{*}{0.9} & 50 & 0.874097 & 0.887479 & 0.857408 & 0.856019 & 0.887182 & 0.884954 & 0.85549 & 0.850641 \\
\hline & 100 & 0.886903 & 0.89931 & 0.876962 & 0.88131 & 0.901159 & 0.897180 & 0.881226 & 0.876444 \\
\hline & 150 & 0.891423 & 0.900933 & 0.885742 & 0.88365 & 0.899921 & 0.901486 & $\mathbf{0 . 8 8 3 9 0 8}$ & 0.884603 \\
\hline \multirow{3}{*}{ - 0.1} & 50 & -0.09419 & -0.09563 & -0.09162 & -0.09205 & -0.09861 & -0.10265 & -0.09167 & -0.09731 \\
\hline & 100 & -0.09339 & -0.10032 & -0.09632 & -0.09598 & -0.10091 & -0.10080 & -0.09800 & -0.09690 \\
\hline & 150 & -0.10044 & -0.10275 & -0.09732 & -0.09662 & -0.10491 & -0.10166 & -0.10042 & -0.09743 \\
\hline \multirow{3}{*}{-0.5} & 50 & -0.48430 & -0.49913 & -0.50035 & -0.50505 & -0.50048 & -0.50339 & -0.50370 & -0.50505 \\
\hline & 100 & -0.48853 & -0.50293 & -0.50126 & -0.50501 & -0.50154 & -0.50047 & -0.50169 & -0.50073 \\
\hline & 150 & -0.49364 & -0.50359 & -0.50214 & -0.50404 & -0.50196 & -0.50279 & -0.50282 & -0.50270 \\
\hline \multirow{3}{*}{ - 0.9} & 50 & -0.87667 & -0.89707 & -0.85501 & -0.85731 & -0.89923 & -0.90089 & -0.85639 & -0.85106 \\
\hline & 100 & -0.88556 & -0.90254 & -0.87390 & -0.87795 & -0.90008 & -0.90312 & -0.87760 & -0.87699 \\
\hline & 150 & -0.89151 & -0.90285 & -0.88445 & -0.88417 & -0.90425 & -0.90352 & -0.88690 & -0.88567 \\
\hline
\end{tabular}

AR(1) جدول (2) يمثل (متوسط مربعات الخطأ Mse) لأنموذج

بعد استخدم طريقة (Burg) للتقدير وحسب طريقة توليد

\begin{tabular}{|c|c|c|c|c|c|c|c|c|c|}
\hline$\phi_{1}$ & sample & SD & $\max$ & mean & med & $\min$ & $\bmod$ & one & zero \\
\hline \multirow{3}{*}{0.1} & 50 & 0.019953 & 0.020020 & 0.020903 & 0.020936 & 0.018143 & 0.020116 & 0.019102 & 0.019811 \\
\hline & 100 & 0.009500 & 0.009232 & 0.009163 & 0.009168 & 0.009637 & 0.009719 & 0.009953 & 0.01004 \\
\hline & 150 & 0.006237 & 0.006929 & 0.00700 & 0.006999 & 0.006903 & 0.006257 & 0.006552 & 0.00660 \\
\hline \multirow{3}{*}{0.5} & 50 & 0.017217 & 0.015313 & 0.014868 & 0.017596 & 0.014626 & 0.014395 & 0.016989 & 0.017588 \\
\hline & 100 & 0.007289 & 0.007112 & 0.008046 & 0.007703 & 0.007879 & 0.007066 & 0.007586 & 0.007712 \\
\hline & 150 & 0.005275 & 0.004769 & 0.005407 & 0.005103 & 0.004666 & 0.004973 & 0.005056 & 0.005115 \\
\hline \multirow{3}{*}{0.9} & 50 & 0.006072 & 0.004399 & 0.007896 & 0.008275 & 0.004103 & 0.00495 & 0.008224 & 0.009728 \\
\hline & 100 & 0.002470 & 0.001624 & 0.003081 & 0.002671 & 0.001453 & 0.002124 & 0.002699 & 0.003431 \\
\hline & 150 & 0.001479 & 0.001154 & 0.001675 & 0.001997 & 0.001243 & 0.001151 & 0.001951 & 0.001888 \\
\hline \multirow{3}{*}{-0.1} & 50 & 0.019227 & 0.020425 & 0.020010 & 0.018687 & 0.020367 & 0.018156 & 0.019910 & 0.018298 \\
\hline & 100 & 0.009829 & 0.009190 & 0.010114 & 0.009750 & 0.009897 & 0.008794 & 0.009670 & 0.009850 \\
\hline & 150 & 0.006678 & 0.007142 & 0.007159 & 0.006521 & 0.006358 & 0.007101 & 0.006529 & 0.006601 \\
\hline \multirow{3}{*}{-0.5} & 50 & 0.014172 & 0.014290 & 0.014070 & 0.013832 & 0.013980 & 0.013114 & 0.012985 & 0.013561 \\
\hline & 100 & 0.006920 & 0.007496 & 0.007434 & 0.006468 & 0.007530 & 0.007318 & 0.007183 & 0.007314 \\
\hline & 150 & 0.005427 & 0.005013 & 0.004855 & 0.005181 & 0.004843 & 0.004729 & 0.005280 & 0.004687 \\
\hline \multirow{3}{*}{-0.9} & 50 & 0.005181 & 0.003141 & 0.008372 & 0.008603 & 0.003019 & 0.002789 & 0.008482 & 0.009473 \\
\hline & 100 & 0.002556 & 0.001527 & 0.003656 & 0.003005 & 0.001855 & 0.001561 & 0.003269 & 0.003128 \\
\hline & 150 & 0.001444 & 0.001014 & 0.001752 & 0.001845 & 0.000916 & 0.001076 & 0.001777 & 0.001710 \\
\hline
\end{tabular}


جدول (3) يمثل مقدرات الأنموذج AR(1 بأستخدم طريقة (OLS) وحسب طريقة توليد أ

\begin{tabular}{|c|c|c|c|c|c|c|c|c|c|}
\hline$\phi_{1}$ & sample & SD & $\max$ & mean & med & $\min$ & mod & one & zero \\
\hline \multirow{3}{*}{0.1} & 50 & 0.070748 & 0.073808 & 0.07109 & 0.071235 & 0.072797 & 0.073253 & 0.069031 & 0.072452 \\
\hline & 100 & 0.083589 & 0.084812 & 0.08386 & 0.08385 & 0.087718 & 0.086 & 0.087672 & 0.090873 \\
\hline & 150 & 0.088584 & 0.095024 & 0.094578 & 0.094586 & 0.091208 & 0.089408 & 0.091895 & 0.092599 \\
\hline \multirow{3}{*}{0.5} & 50 & 0.436185 & 0.443144 & 0.438039 & 0.435481 & 0.444833 & 0.447636 & 0.437058 & 0.435408 \\
\hline & 100 & 0.472446 & 0.468124 & 0.470094 & 0.466761 & 0.469493 & 0.474511 & 0.466625 & 0.466715 \\
\hline & 150 & 0.480536 & 0.47976 & 0.481015 & 0.478976 & 0.479709 & 0.482357 & 0.479011 & 0.479018 \\
\hline \multirow{3}{*}{0.9} & 50 & 0.796304 & 0.803361 & 0.787312 & 0.786241 & 0.804633 & 0.803477 & 0.785904 & 0.784782 \\
\hline & 100 & 0.850225 & 0.854481 & 0.845234 & 0.850371 & 0.857062 & 0.854349 & 0.850278 & 0.846322 \\
\hline & 150 & 0.867742 & 0.870223 & 0.867068 & 0.865147 & 0.870271 & 0.871539 & 0.865175 & 0.865858 \\
\hline \multirow{3}{*}{-0.1} & 50 & -0.11026 & -0.10746 & -0.10947 & -0.10777 & -0.11103 & -0.11293 & -0.10800 & -0.11360 \\
\hline & 100 & -0.10118 & -0.10533 & -0.10465 & -0.10477 & -0.10534 & -0.10555 & -0.10554 & -0.10520 \\
\hline & 150 & -0.10571 & -0.10569 & -0.10308 & -0.10229 & -0.10775 & -0.10513 & -0.10573 & -0.10287 \\
\hline \multirow{3}{*}{-0.5} & 50 & -0.48741 & -0.48076 & -0.48009 & -0.48580 & -0.48032 & -0.48355 & -0.48489 & -0.48518 \\
\hline & 100 & -0.48975 & -0.49016 & -0.48907 & -0.49173 & -0.48926 & -0.48798 & -0.48881 & -0.48821 \\
\hline & 150 & -0.49466 & -0.49361 & -0.49212 & -0.49429 & -0.49196 & -0.49282 & -0.49294 & -0.49272 \\
\hline \multirow{3}{*}{-0.9} & 50 & -0.85451 & -0.85945 & -0.84803 & -0.85006 & -0.86396 & -0.86508 & -0.84648 & -0.84336 \\
\hline & 100 & -0.87413 & -0.87858 & -0.87048 & -0.87413 & -0.87629 & -0.87884 & -0.87258 & -0.87310 \\
\hline & 150 & -0.88374 & -0.88338 & -0.88196 & -0.88180 & -0.88554 & -0.88467 & -0.88387 & -0.88346 \\
\hline
\end{tabular}

جدول (4) يمثل (متوسط مربعات الخطأ Mse) لأنموذج AR(1) بعد استخدم طريقة (OLS) للتقير وحسب طريقة توليد

\begin{tabular}{|c|c|c|c|c|c|c|c|c|c|}
\hline$\phi_{1}$ & sample & SD & $\max$ & mean & med & $\min$ & $\bmod$ & one & Zero \\
\hline \multirow{3}{*}{0.1} & 50 & 0.020178 & 0.018687 & 0.021441 & 0.021465 & 0.017328 & 0.018505 & 0.019553 & 0.019832 \\
\hline & 100 & 0.009456 & 0.008944 & 0.009436 & 0.009441 & 0.008981 & 0.009106 & 0.010077 & 0.010118 \\
\hline & 150 & 0.006339 & 0.006564 & 0.006952 & 0.00695 & 0.006633 & 0.006085 & 0.006521 & 0.006624 \\
\hline \multirow{3}{*}{0.5} & 50 & 0.020762 & 0.018103 & 0.019117 & 0.021093 & 0.017756 & 0.01698 & 0.020477 & 0.021101 \\
\hline & 100 & 0.008094 & 0.007871 & 0.008702 & 0.008514 & 0.008835 & 0.007996 & 0.008434 & 0.008518 \\
\hline & 150 & 0.005578 & 0.005216 & 0.005842 & 0.005578 & 0.005059 & 0.005283 & 0.005544 & 0.005589 \\
\hline \multirow{3}{*}{0.9} & 50 & 0.019146 & 0.017022 & 0.022006 & 0.021888 & 0.016702 & 0.017504 & 0.022274 & 0.022891 \\
\hline & 100 & 0.005528 & 0.004749 & 0.00643 & 0.005861 & 0.004644 & 0.004974 & 0.005805 & 0.006278 \\
\hline & 150 & 0.002807 & 0.002497 & 0.00288 & 0.003249 & 0.002569 & 0.002433 & 0.003231 & 0.003099 \\
\hline \multirow{3}{*}{-0.1} & 50 & 0.018819 & 0.018103 & 0.019400 & 0.0182312 & 0.017461 & 0.016164 & 0.019067 & 0.018410 \\
\hline & 100 & 0.009568 & 0.008555 & 0.009978 & 0.0096154 & 0.009343 & 0.008080 & 0.009464 & 0.009702 \\
\hline & 150 & 0.006633 & 0.006779 & 0.007062 & 0.0065228 & 0.005972 & 0.006732 & 0.006438 & 0.006518 \\
\hline \multirow{3}{*}{-0.5} & 50 & 0.013357 & 0.013411 & 0.013497 & 0.012829 & 0.013374 & 0.012521 & 0.012060 & 0.012819 \\
\hline & 100 & 0.006706 & 0.007163 & 0.007191 & 0.006189 & 0.007278 & 0.007054 & 0.006963 & 0.007025 \\
\hline & 150 & 0.005292 & 0.004876 & 0.004768 & 0.005027 & 0.004759 & 0.004639 & 0.005156 & 0.004590 \\
\hline \multirow{3}{*}{ - 0.9} & 50 & 0.007106 & 0.006004 & 0.009108 & 0.009305 & 0.005405 & 0.004989 & 0.009567 & 0.010314 \\
\hline & 100 & 0.003231 & 0.002441 & 0.003883 & 0.003232 & 0.002840 & 0.002437 & 0.003582 & 0.003336 \\
\hline & 150 & 0.001669 & 0.001533 & 0.001854 & 0.001935 & 0.001329 & 0.001542 & 0.001888 & 0.001801 \\
\hline
\end{tabular}


جدول (5) يمثل مقدرات الأنموذج (MR(1) بأستخدم طريقة (MLE) وحسب طريقة توليد

\begin{tabular}{|c|c|c|c|c|c|c|c|c|c|}
\hline$\phi_{1}$ & sample & SD & $\max$ & mean & med & $\min$ & $\bmod$ & one & Zero \\
\hline \multirow{3}{*}{0.1} & 50 & 0.11828 & 0.12859 & 0.11958 & 0.11972 & 0.12322 & 0.12826 & 0.11637 & 0.11881 \\
\hline & 100 & $\mathbf{0 . 1 1 7 2 7}$ & 0.12500 & 0.11251 & 0.11252 & 0.12577 & 0.13190 & 0.12426 & 0.12248 \\
\hline & 150 & 0.11791 & 0.13575 & 0.12192 & 0.12192 & 0.13098 & 0.13153 & 0.11326 & 0.11923 \\
\hline \multirow{3}{*}{0.5} & 50 & 0.47303 & 0.51831 & 0.46884 & 0.46879 & 0.51840 & 0.52134 & 0.47664 & 0.46820 \\
\hline & 100 & $\mathbf{0 . 4 7 9 7 5}$ & 0.52981 & $\mathbf{0 . 4 6 6 9 7}$ & 0.46881 & 0.53038 & 0.53295 & 0.47556 & 0.46837 \\
\hline & 150 & 0.47609 & 0.53900 & 0.47147 & 0.47081 & 0.54058 & 0.54423 & 0.47850 & 0.47072 \\
\hline \multirow{3}{*}{0.9} & 50 & $\mathbf{0 . 8 8 1 0 7}$ & 0.90018 & $\mathbf{0 . 8 5 2 8 6}$ & 0.85266 & 0.90014 & 0.89896 & 0.85113 & 0.84422 \\
\hline & 100 & 0.88260 & 0.91965 & 0.85024 & 0.85453 & 0.91971 & 0.91583 & 0.85521 & 0.84631 \\
\hline & 150 & 0.88684 & 0.92828 & 0.85245 & 0.85011 & 0.92824 & 0.92913 & 0.85179 & 0.84784 \\
\hline \multirow{3}{*}{-0.1} & 50 & -0.11704 & -0.12561 & $\begin{array}{l}-0.11468 \\
\end{array}$ & -0.11406 & -0.12842 & -0.12736 & -0.11580 & -0.11741 \\
\hline & 100 & -0.10408 & -0.11249 & -0.10708 & -0.10560 & -0.11358 & -0.11238 & -0.10833 & -0.10718 \\
\hline & 150 & -0.10548 & -0.11012 & -0.10342 & -0.10238 & -0.11188 & -0.11050 & -0.10574 & -0.10286 \\
\hline \multirow{3}{*}{-0.5} & 50 & -0.48121 & -0.51940 & -0.52105 & -0.52549 & -0.52127 & -0.52397 & -0.52388 & -0.52558 \\
\hline & 100 & -0.48728 & -0.51619 & -0.51437 & -0.51879 & -0.51462 & -0.51348 & -0.51507 & -0.51370 \\
\hline & 150 & -0.49285 & -0.51401 & -0.51243 & -0.51441 & -0.51226 & -0.51298 & -0.51304 & -0.51287 \\
\hline \multirow{3}{*}{-0.9} & 50 & -0.88303 & -0.91603 & -0.84757 & -0.85028 & -0.91621 & -0.91825 & -0.85208 & -0.84468 \\
\hline & 100 & -0.88906 & -0.91767 & -0.86988 & -0.87423 & -0.91510 & -0.91857 & -0.87486 & -0.87337 \\
\hline & 150 & -0.89393 & -0.91662 & -0.88176 & -0.88142 & -0.91719 & -0.91668 & -0.88476 & -0.88276 \\
\hline
\end{tabular}

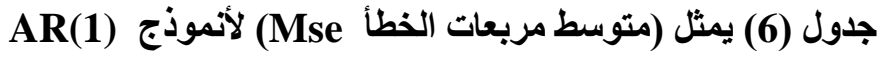

بعد استخدم طريقة (MLE) للتقدير وحسب طريقة توليد

\begin{tabular}{|c|c|c|c|c|c|c|c|c|c|}
\hline$\phi_{1}$ & sample & SD & $\max$ & mean & med & $\min$ & $\bmod$ & one & zero \\
\hline \multirow{3}{*}{0.1} & 50 & 0.011586 & 0.013957 & 0.012012 & 0.012042 & 0.012363 & 0.013624 & 0.011369 & 0.011430 \\
\hline & 100 & 0.011269 & 0.012981 & 0.010503 & 0.010506 & 0.012673 & 0.013761 & 0.012012 & 0.012261 \\
\hline & 150 & 0.010564 & 0.014811 & 0.011614 & 0.011616 & 0.014431 & 0.012959 & 0.011148 & 0.011389 \\
\hline \multirow{3}{*}{0.5} & 50 & 0.017172 & 0.016446 & 0.014810 & 0.017574 & 0.015559 & 0.015345 & 0.016914 & 0.017594 \\
\hline & 100 & 0.015512 & 0.016042 & 0.015929 & 0.016651 & 0.016407 & 0.016291 & 0.016147 & 0.016712 \\
\hline & 150 & 0.014692 & 0.014842 & 0.017050 & 0.015140 & 0.015639 & 0.015385 & 0.014650 & 0.015179 \\
\hline \multirow{3}{*}{0.9} & 50 & 0.005448 & 0.003884 & 0.008030 & 0.008203 & 0.003453 & 0.004046 & 0.008313 & 0.010375 \\
\hline & 100 & 0.005417 & 0.002951 & 0.008847 & 0.008142 & 0.002667 & 0.003217 & 0.007943 & 0.010070 \\
\hline & 150 & 0.004119 & 0.002613 & 0.007927 & 0.008429 & 0.002569 & 0.002599 & 0.008188 & 0.009078 \\
\hline \multirow{3}{*}{-0.1} & 50 & 0.011346 & 0.013533 & 0.011569 & 0.010969 & 0.013840 & 0.012775 & 0.011749 & 0.010993 \\
\hline & 100 & 0.006691 & 0.007122 & 0.006915 & 0.006902 & 0.007733 & 0.006893 & 0.006858 & 0.006821 \\
\hline & 150 & 0.005341 & 0.006095 & 0.005470 & 0.004936 & 0.005434 & 0.005676 & 0.005178 & 0.005076 \\
\hline \multirow{3}{*}{-0.5} & 50 & 0.013926 & 0.015277 & 0.014931 & 0.014945 & 0.014906 & 0.014175 & 0.014033 & 0.014674 \\
\hline & 100 & 0.006907 & 0.007973 & 0.007838 & 0.006969 & 0.007946 & 0.007710 & 0.007588 & 0.007721 \\
\hline & 150 & 0.005434 & 0.005274 & 0.005123 & 0.005456 & 0.005103 & 0.004963 & 0.005528 & 0.004927 \\
\hline \multirow{3}{*}{-0.9} & 50 & 0.004715 & 0.002528 & 0.009005 & 0.009169 & 0.002435 & 0.002406 & 0.008702 & 0.010111 \\
\hline & 100 & 2322 & 0.001506 & 3851 & 0.003159 & 0.001771 & 0.001583 & 0.003357 & 0.003295 \\
\hline & 150 & 0.001410 & 0.001118 & 0.001835 & 0.001930 & 0.001056 & 0.001165 & 0.001811 & 0.001780 \\
\hline
\end{tabular}




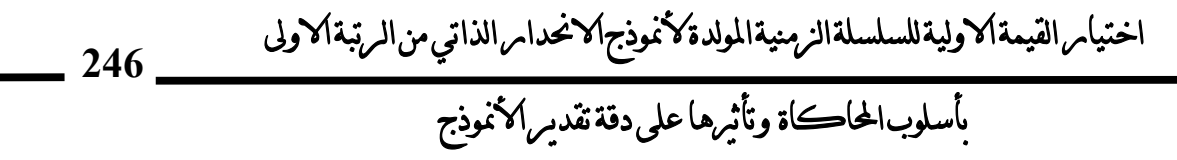

5- تحليل النتائج

من اجل بيـان أفضل طريقة من طرائق حسـاب القيم الابتدايـة للمشـاهدة (

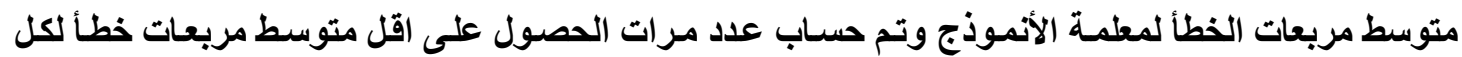

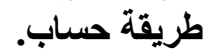

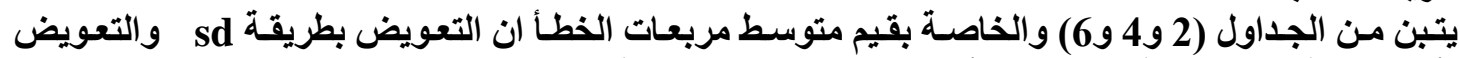

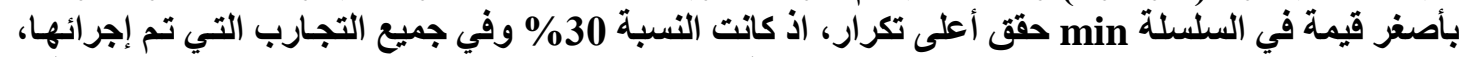

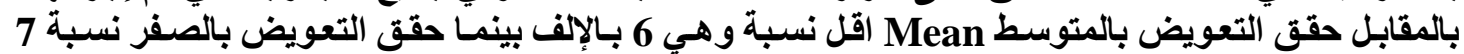
بالالف \% والجدول (7) يبين هذه النتائج.

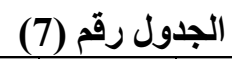

\begin{tabular}{|c|c|c|}
\hline & ط العدية & $\begin{array}{c}\text { ط التعويضة } \\
\text { النسبة }\end{array}$ \\
\hline 0.11 & 6 & SD \\
\hline 0.06 & 3 & mean \\
\hline 0.30 & 16 & min \\
\hline 0.26 & 14 & mod \\
\hline 0.07 & 4 & one \\
\hline 0.06 & 3 & zero \\
\hline 0.07 & 4 & max \\
\hline 0.07 & 4 & median \\
\hline
\end{tabular}

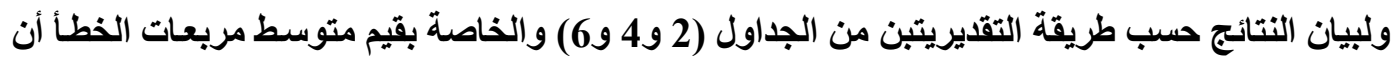

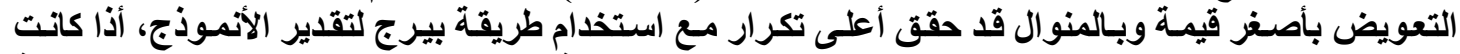

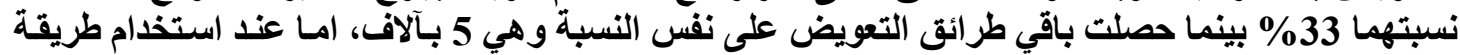

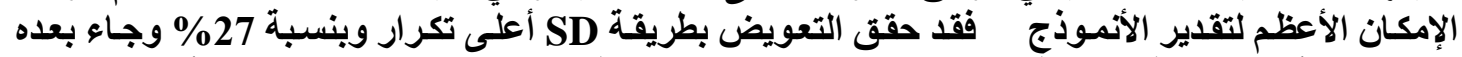

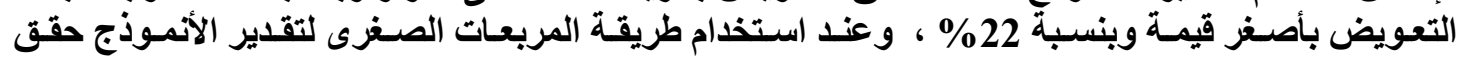

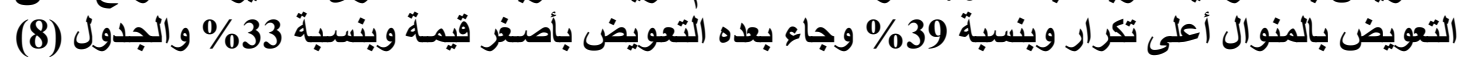
يبين هذه النتائج. 


\section{$-247$

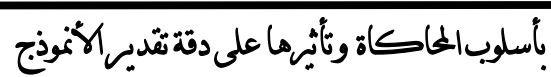

جدول رقم (8) يوضح طريقة التقديرمع طريقة التعويض

\begin{tabular}{|c|c|c|c|c|c|c|}
\hline \multicolumn{2}{|c|}{ OLS } & \multicolumn{2}{|c|}{ MLE } & \multicolumn{2}{|c|}{ Burg } & \multirow{2}{*}{ طريقة التقدير التعويض } \\
\hline النسبة & التكرار & النسبة & التكرار & النسبة & التكرار & \\
\hline 0.00 & $\mathbf{0}$ & 0.28 & 5 & 0.06 & 1 & SD \\
\hline 0.00 & 0 & 0.11 & 2 & 0.06 & 1 & mean \\
\hline $\mathbf{0 . 3 3}$ & 6 & 0.22 & 4 & $\mathbf{0 . 3 3}$ & 6 & $\min$ \\
\hline 0.39 & 7 & 0.06 & 1 & 0.33 & 6 & $\bmod$ \\
\hline 0.06 & 1 & 0.11 & 2 & 0.06 & 1 & one \\
\hline 0.06 & 1 & 0.06 & 1 & 0.06 & 1 & zero \\
\hline 0.11 & 2 & 0.06 & 1 & 0.06 & 1 & $\max$ \\
\hline 0.06 & 1 & 0.11 & 2 & 0.06 & 1 & median \\
\hline
\end{tabular}

ولبيان النتائج حسب حجم العينة يتبن من الجداول (2 و4 و6) و والخاصة بقيم متوسط مربعات الخطأ أن

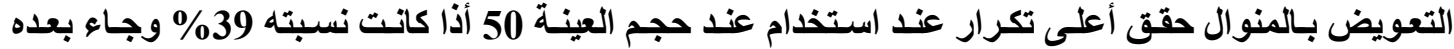

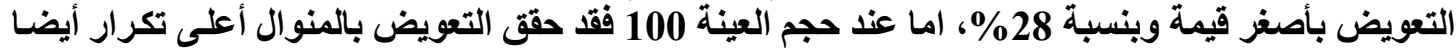

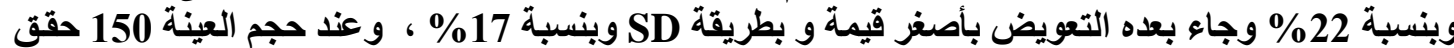
التعويض بأصغر قيمة اعلى تكرار وبنسبة 44\% بونة وجاء بعده التعويض بالمنوال وبنسبة 17\% و 17 والجدول (9) يبين هذه النتائج. الجدول (9) يوضح طريقة التعويض وحجم العينة

\begin{tabular}{|c|c|c|c|c|c|c|}
\hline \multicolumn{2}{|c|}{ Sample $=150$} & \multicolumn{2}{|c|}{ Sample $=100$} & \multicolumn{2}{|c|}{ Sample $=50$} & \multirow[b]{2}{*}{ الطريقة } \\
\hline النسبة & التكرار & النسبة & التكرار & النسبة & التكرار & \\
\hline 0.11 & 2 & 0.17 & 3 & 0.06 & 1 & SD \\
\hline 0.00 & $\mathbf{0}$ & 0.11 & 2 & 0.06 & 1 & mean \\
\hline 0.44 & 8 & 0.17 & 3 & 0.28 & 5 & $\min$ \\
\hline 0.17 & 3 & 0.22 & 4 & 0.39 & 7 & mode \\
\hline 0.06 & 1 & 0.00 & $\mathbf{0}$ & 0.17 & 3 & one \\
\hline 0.17 & 3 & 0.00 & $\mathbf{0}$ & 0.00 & $\mathbf{0}$ & zero \\
\hline 0.00 & $\mathbf{0}$ & 0.22 & 4 & 0.00 & $\mathbf{0}$ & $\max$ \\
\hline 0.06 & 1 & 0.11 & 2 & 0.06 & 1 & median \\
\hline
\end{tabular}




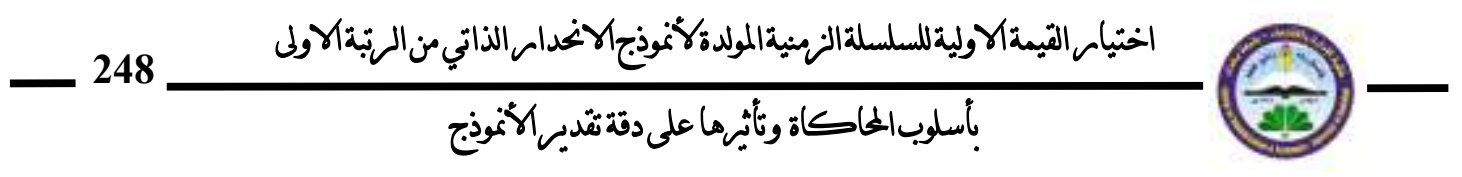
6- - الاستنتاجات و التوصيات

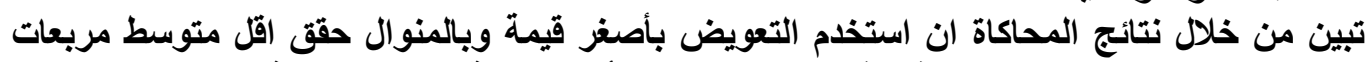

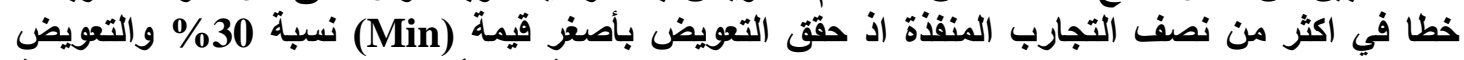

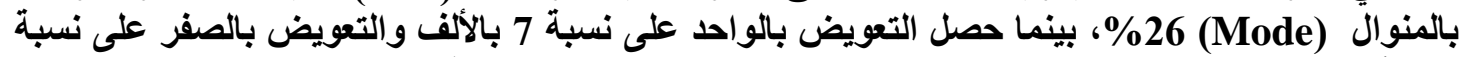

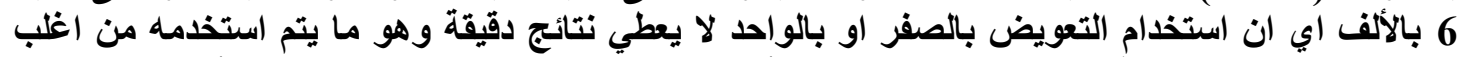

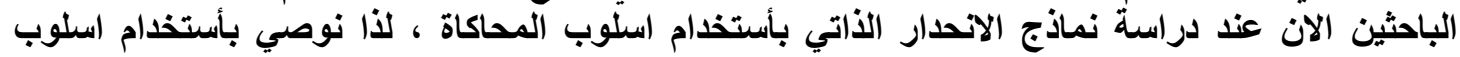
تعويض القيم الابتداية للمشاهدة ( النظرعن طريقة التقدير وحجم العينة

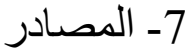

[1] Aydin,S., " Determination of autoregressive model orders for seizure detection" Turk J Elec Eng \& Comp Sci, Vol.18, No.1, 2010

[2] Hamilton, J., " Time series Analysis," New Jersey , 1994

[3] MAKHOUL , J.;"Stable and Efficient Lattice Methods for Linear Prediction" ; IEEE Transaction on Acoustics, Speech, and Signal Processing, Vol, ASSP- 25, NO. 5 , October 1977

[4] Roth,K., \& Kauppinen,I., " FREQUENCYWARPED BURG'S METHOD FOR ARMODELING " IEEE Workshop on Applications of Signal Processing to Audio and Acoustics, October 19-22, 2003,

[5] Steven M.KAY "Efficient Generation of Colored Noise" Proceedings of the IEEE, Vol.4, April 1981

[6] Tsay,R., " Analysis of Financial Time Series," John Wiley \& Sons, 